\title{
A RADIATION-CONDITIONED GAUSSIAN INDUCED MGD CONEFIELD*
}

\author{
BY \\ LIM CHEE-SENG** \\ University of Calgary, Alberta
}

Summary. Explicit radiation-conditioned solutions are derived for magneticallyaligned MGD flow past a Gaussian source. If the latter's principal dimension is small, then corresponding to a supersonic-superAlfvénic (or restricted subsonic-subAlfvénic) flow, a strong perturbation field is encountered downstream from a downstream (or upstream from an upstream) tangent cone to the "fast" sheet (or one of the two "slow" cusped sheets) of the Friedrichs wavefront, while a weak estimable field appears upstream (or downstream) from this cone. The strong field is represented by an expansion with accessible asymptotic development. For a finite principal source dimension, other asymptotic modes can be extracted from a separate uniformly valid formulation. In particular, the asymptotic behavior near the dividing tangent cone is nonsingular and suggests the possible existence there of a conical sheath within which the field is probably most concentrated, and across which its transition from strong to weak occurs gradually.

1. Introduction. One of the basic three-dimensional MGD flow problems is that attempted by Crapper [4], viz. the arbitrarily directed flow past a source with the Gaussian strength

$$
(l \sqrt{ } \pi)^{-3} \exp \left(-\mathbf{x}^{2} / l^{2}\right)
$$

Using Lighthill's ([7; see also $[8,9])$ stationary-phase-cum-radiation condition technique, Crapper achieved an asymptotic far-field solution valid for any moderate principal dimension $l$. Because of the complicated dependence on geometrically involved parameters, a certain degree of explicitness is unavoidably sacrificed.

The purpose of this paper is to investigate more closely the special case corresponding to a magnetically aligned, supersonic-superAlfvénic flow (which overlaps Crapper's cursory treatment of the flow at Mach number 3 and Alfvén number 4), as well as restricted subsonic-subAlfvénic flow (avoided by Crapper), and, particularly, to obtain explicit radiation-conditioned results that (i) hold for any observation position but small $l$, and (ii) evolve asymptotically, for finite $l$, from a uniform representation. Since expression (1.1) approaches the Dirac delta function $\delta(\mathbf{x})$ as $l \rightarrow 0$, a direct byproduct associated with (i) is the exact Green's function. One of the various asymptotic modes parallels Crapper's; though there is general agreement, a certain discrepancy also emerges.

Perturbations permeate the entire fluid space and would be, but for a complete synthesis, traversed by a pair of covertexed cones. However, the applied radiation condi-

\footnotetext{
${ }^{*}$ Received August 4, 1976. Portions of this work were completed under a Visiting Research Associateship at the University of Calgary. I would like to thank Professors Peter Lancaster, Samir Majumdar and D. Rex Westbrook for their hospitality and the support derived from their N.R.C. (Canada) operating grants.

** Permanent address: Department of Mathematics, University of Singapore, Singapore.
} 
tion admits only one member (of this pair) which restricts a strong field to one side, and a weak field to the other side. Such cones are amongst those qualitatively studied by Cumberbatch [5]. They feature primarily in the point disturbance theory, wherein certain phenomena may be anticipated for aligned flow by analogy with conventional gas dynamics (see e.g. [10]). This is especially so in two-dimensional theory, but not quite with threedimensional problems; for example, in the restricted subsonic-subAlfvénic regime, the perturbation confinement zone has never been convincingly established although the confining conical boundary is believed, on purely physical grounds, to project upstream. Part of the argument relies intuitively on a physically conceived Huyghens-oriented principle adopted by Cumberbatch [5]. Instead, our construction will be based on a combination of Fourier and geometrical inversion, plus the radiation condition effect.

2. Transformation of equations of motion. Consider a uniform state wherein an unbounded mass of perfectly conducting, inviscid, compressible fluid with density $\rho_{0}$ and sound speed $c$ is moving at velocity $\mathbf{V}$ through a magnetic field $\mathbf{H}$. Let a denote the Alfvén velocity (parallel to $\mathbf{H}$ ), and $a=|\mathbf{a}|$. Small disturbances are being induced by a weak fluid injecting source with productivity rate function $S$. Suppose corresponding perturbations in the magnetic field, the fluid velocity and pressure are, respectively, $|\mathbf{H}| \mathbf{h}, \mathbf{v}, \rho_{0} p$. The linearized equations of continuity, induction, momentum and divergence are then

$$
\begin{gathered}
D p / D t+c^{2} \nabla \cdot \mathbf{v}=c^{2} S, \quad \partial \mathbf{h} / \partial t=\nabla \times\left(\mathbf{v} \times \mathbf{a} a^{-1}\right)+\nabla \times(\mathbf{V} \times \mathbf{h}), \\
D \mathbf{v} / D t+\nabla p=a(\nabla \times \mathbf{h}) \times \mathbf{a}, \quad \nabla \cdot \mathbf{h}=0,
\end{gathered}
$$

where $D / D t \equiv \partial / \partial t+\mathbf{V} \cdot \nabla$. We next define a scalar $P$ and a vector $\mathbf{Q}$ via the differential equations

$$
\begin{gathered}
{\left[\left(M^{2}+A^{2}\right)(D / D t)^{2}-(\mathbf{V} \cdot \nabla)^{2}\right](p+a \mathbf{a} \cdot \mathbf{h})=\left[A^{2}(D / D t)^{2}-(\mathbf{V} \cdot \nabla)^{2}\right] P,} \\
{\left[\left(M^{2}+A^{2}\right)(D / D t)^{2}-(\mathbf{V} \cdot \nabla)^{2}\right][a(\mathbf{a} \cdot \nabla) \mathbf{h}-D \mathbf{v} / D t]} \\
=\left[A^{2}(D / D t)^{2}-(\mathbf{V} \cdot \nabla)^{2}\right] D \mathbf{Q} / D t,
\end{gathered}
$$

where $M=M(V)=V / c$ (Mach number) and $A=A(V)=V / a$ (Alfvén number), with $V$ $=|\mathbf{V}|$.

With reference to the $\mathbf{x}=(x, y, z)$ coordinate frame, let $\mathbf{a}=(a 0,0)$.

We assume a magnetically aligned flow: $\mathbf{V}=(V, 0,0)$. To attain an ultimate steady state, it is normally necessary that the source act steadily. The radiation condition may now be accommodated, following Lighthill $[7,8,9]$, by first envisaging a certain unsteady development, subsequent to the source function $S$ acquiring an exponential growth to become

$$
S \exp (\epsilon t)=\exp (\epsilon t) \int_{-\infty}^{\infty} \int_{-\infty}^{\infty} \int_{-\infty}^{\infty} \hat{S}(\boldsymbol{\alpha}) \exp (i \boldsymbol{\alpha} \cdot \mathbf{x}) d \alpha d \beta d \gamma,
$$

in terms of its Fourier transform $S=S(\alpha)$ (signified by^), dependent on the wave vector $\alpha$ $=(\alpha, \beta, \gamma)$ :

$$
S(\boldsymbol{\alpha})=(2 \pi)^{-3} \int_{-\infty}^{\infty} \int_{-\infty}^{\infty} \int_{-\infty}^{\infty} S \exp (-i \boldsymbol{\alpha} \cdot \mathbf{x}) d x d y d z
$$

Throughout, $\epsilon>0$. The radiation condition is then satisfied if perturbations are likewise allowed to grow exponentially: e.g., 


$$
\mathbf{v}=\exp (\epsilon t) \int_{-\infty}^{\infty} \int_{-\infty}^{\infty} \int_{-\infty}^{\infty} \hat{\mathbf{v}}(\boldsymbol{\alpha}) \exp (i \boldsymbol{\alpha} \cdot \mathbf{x}) d \alpha d \beta d \gamma
$$

By the time free perturbations coming in from infinity infiltrate our observation region, they are negligibly small compared with source-emitted $O(\exp \epsilon t)$ quantities, and therefore tend to escape detection near the steady state.

Fourier transforming (2.1) and (2.2), noting that $D / D t$ converts into $i \alpha V_{\epsilon}$ with $V_{\epsilon}=V$ $-i \epsilon \alpha^{-1}$, it then follows from elementary vector manipulations that

$$
\begin{gathered}
\alpha M_{\epsilon}^{2} \hat{p}+V_{\epsilon} \boldsymbol{\alpha} \cdot \hat{\mathbf{v}}=-i V_{\epsilon} \hat{S}, \\
\alpha V_{\epsilon} \hat{\mathbf{h}}+(\alpha \cdot \hat{\mathbf{v}}, 0,0)=\alpha \hat{\mathbf{v}}, \\
\alpha\left(a^{2} \hat{\mathbf{h}}-V_{\epsilon} \hat{\mathbf{v}}\right)=\alpha\left(\hat{p}+a^{2} \hat{h}_{1}\right), \\
\alpha \cdot \hat{\mathbf{h}}=0,
\end{gathered}
$$

where $h_{1}$ is the $x$-component of $\mathbf{h}, M_{\epsilon}=M\left(V_{\epsilon}\right)=V_{\epsilon} / c$. Let $A_{\epsilon}=A\left(V_{\epsilon}\right)=V_{\epsilon} / a$. Then (2.3) and (2.4) transform into

$$
\hat{p}+a^{2} \hat{h_{1}}=\frac{\left(A_{\epsilon}{ }^{2}-1\right) \hat{P}}{M_{\epsilon}{ }^{2}+A_{\epsilon}{ }^{2}-1}, \quad a^{2} \hat{h}-V_{\epsilon} \hat{\mathrm{v}}=\frac{V_{\epsilon}\left(A_{\epsilon}{ }^{2}-1\right) \mathbf{Q}}{M_{\epsilon}{ }^{2}+A_{\epsilon}{ }^{2}-1},
$$

whence $(2.10)$ is equivalent to

$$
\alpha \mathrm{Q} V_{\epsilon}=\alpha \hat{P} .
$$

Furthermore, it can be shown from (2.8), the $x$-components of (2.9) and (2.10), as well as $(2.11)$ and (2.12), that

$$
\alpha \cdot Q V_{\epsilon}=\alpha \hat{P} \lambda^{2}\left(V-i \epsilon \alpha^{-1}\right)+i V \hat{\epsilon},
$$

$\lambda=\lambda(V)$ being a magnetoacoustic flow number defined by

$$
\lambda^{-2}=M^{-2}+A^{-2}-M^{-2} A^{-2} .
$$

Hence, solving (2.13) and (2.14) for $\hat{P}$ and $Q$, we get:

$$
\hat{P}=\frac{i \alpha V_{\epsilon} S(\alpha)}{\alpha^{2}-\alpha^{2} \lambda^{2}\left(V-i \epsilon \alpha^{-1}\right)}, \quad \mathrm{Q}=\frac{i \alpha S(\alpha)}{\alpha^{2}-\alpha^{2} \lambda^{2}\left(V-i \epsilon \alpha^{-1}\right)} .
$$

3. Radiation-conditioned inversion. The transforms in (2.16) must be inverted to recover $P$ and $\mathbf{Q}$. It is seen that

$$
P=D \Phi / D t, \quad \mathbf{Q}=\nabla \Phi,
$$

if

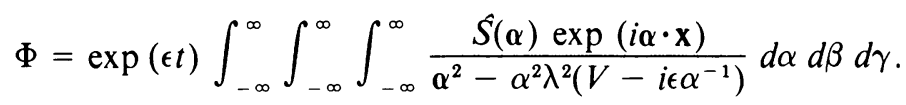

We now assume that the Gaussian of (1.1) represents $S$. Then

$$
S(\alpha)=(2 \pi)^{-3} \exp \left(-1 / 4, \alpha^{2} l^{2}\right) .
$$

Whereupon, introducing cylindrical polar coordinates, $(x, r, \theta)$ and $(\alpha, \kappa, \omega)$ with $y=r$ $\cos \theta, z=r \sin \theta, \beta=\kappa \cos \omega, \gamma=\kappa \sin \omega$, and employing [11 (6) §3.3] 


$$
\int_{\theta-\pi}^{\theta+\pi} \exp [i \kappa r \cos (\omega-\theta)] d \omega=2 \int_{0}^{\pi} \exp (i \kappa r \cos \omega) d \omega=2 \pi J_{0}(\kappa r)
$$

(the Bessel function of order zero), we reduce (3.2) to the axisymmetric form

$$
\Phi=\exp (\epsilon t)(4 \pi)^{-1} \int_{0}^{\infty} J_{0}(\kappa r) \exp \left(-\frac{1}{4} \kappa^{2} l^{2}\right)[\chi(x \mid \kappa)-\chi(x \mid-\kappa)] d \kappa,
$$

with

$$
\chi(x \mid \kappa)=(2 \pi)^{-1} \int_{-\infty}^{\infty} \frac{\exp \left(i \alpha x-\frac{1}{4} \alpha^{2} l^{2}\right) d \alpha}{\kappa+\alpha\left[\lambda^{2}\left(V-i \epsilon \alpha^{-1}\right)-1\right]^{1 / 2}} .
$$

Crapper's paper [4] focuses on a velocity gradient comparable with a second derivative of our function $\Phi$. Moreover, as the mathematical derivation adheres to an arbitrarily directed flow, his perturbation field is not generally axisymmetric. So, unlike (3.5) with (3.6), his initial representation remains a triple integral, and is resolved by a threedimensional stationary phase method which we will not adopt.

However, Crapper's arguments do incorporate Lighthill's radiation condition technique. In the present analysis, this may be completed by determining $\Phi$ from (3.5) and (3.6), and then letting $\epsilon \rightarrow 0$. What remains constitutes the desired steady-state solution. Before taking the limit, however, evaluation can be done through linear approximations for small $\epsilon$. Thus, in particular,

$$
\kappa+\alpha\left[\lambda^{2}\left(V-i \epsilon \alpha^{-1}\right)-1\right]^{1 / 2} \approx\left(\lambda^{2}-1\right)^{1 / 2}\left[\alpha-i \epsilon \Omega+\kappa\left(\lambda^{2}-1\right)^{-1 / 2}\right]
$$

where

$$
\Omega=\lambda(V) \lambda^{\prime}(V)\left[\lambda^{2}(V)-1\right]^{-1}=\frac{M^{2} A^{2}\left(M^{2}+A^{2}-2\right)}{V\left(\lambda^{2}-1\right)\left(M^{2}+A^{2}-1\right)^{2}},
$$

via (2.15). Note that

$$
\lambda^{2}-1=\left(M^{2}-1\right)\left(A^{2}-1\right)\left(M^{2}+A^{2}-1\right)^{-1} .
$$

The right side of (3.7) vanishes at

$$
\alpha=i \epsilon \Omega-\kappa\left(\lambda^{2}-1\right)^{-1 / 2},
$$

which, with reference to (3.6), is a simple pole of the approximated integrand. The latter stays analytic elsewhere.

Hereafter, we keep to a hyperbolic flow regime [10]: $\lambda>1$. This is equivalent, by virtue of (3.9), to either

$$
M>1 \text { and } A>1 \text { (a supersonic-superAlfvénic stream), }
$$

or

$M<1$ and $A<1$, but $M^{2}+A^{2}>1$ (a restricted subsonic-subAlfvénic stream).

The pole of (3.10) is therefore predominantly real, but slightly displaced into the complex half-plane $\operatorname{Im} \alpha \gtrless 0$ whenever $\Omega \gtrless 0$, i.e., in view of (3.8), depending on whether (3.11) or (3.12) holds.

The integral $\chi(x \mid \kappa)$ may now be considered via contour integration. The usual appeal to Jordan's lemma fails because the integrand factor $\exp \left(-1 / 4 \alpha^{2} l^{2}\right)$ diverges as $|\alpha| \rightarrow \infty$ 
throughout either of the two sectors: $\pi / 4<|\arg \alpha|<3 \pi / 4$. Instead, we shall evaluate $\chi(x \mid \kappa)$ to an estimable error corresponding to a significantly small $l$ and, tentatively, for $x$ $\neq 0$. First deform the real interval $\left(-N / l^{2}, N / l^{2}\right)$, chosen to satisfy $N>\kappa l^{2}\left(\lambda^{2}-1\right)^{-1 / 2}$ for each $\kappa \in(0, \infty)$. Suppose the deformation is rectangular and comprises the horizontal path

$$
\mathfrak{L}: \alpha=\operatorname{Re} \alpha+i 2 x / l^{2} \quad\left(-N / l^{2}<\operatorname{Re} \alpha<N / l^{2}\right)
$$

joined to the specified interval via the two vertical portions

$$
\mathfrak{L}_{ \pm}: \alpha= \pm N / l^{2}+i 2 x s / l^{2} \quad(0<s<1) .
$$

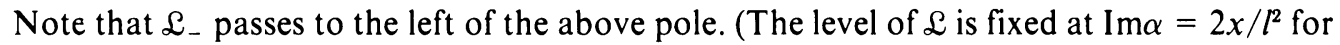
a special reason. Suppose it is assigned along $\operatorname{Im} \alpha=2 x_{0} / l^{2}$ instead. Then the integrand in (3.6), when integrated along $\mathcal{L}$, imparts a controlling factor, viz. $\exp \left[-x_{0} l^{-2}\left(2 x-x_{0}\right)\right]$, which must stay bounded as $l \rightarrow 0$. This is clearly satisfied by the chosen level, i.e. with $x_{0}$ $=x$.) When $x \gtrless 0$, the deformed contour (see Fig. 1) is \pm clockwise directed into the region Im $\alpha \gtrless 0$ and has (or has not) therefore crossed the solitary pole provided $\Omega \gtrless 0$ (or $\Omega \lessgtr 0$ ). For a full crossing, it is understood that we already have $\epsilon<2|x| / I^{2}|\Omega|$. Consequently, according to residue theory,

$\int_{-N / l^{2}}^{N / l^{2}}=2 \pi i(\operatorname{sgn} x) H(x \Omega)\left[\right.$ residue at $\left.\alpha=i \epsilon \Omega-\kappa\left(\lambda^{i}-1\right)^{-1 / 2}\right]+\int_{L^{2}}+\int_{\mathscr{L}_{-}}+\int_{\mathscr{L}_{+}}$,

where each integ, al, as well as the stipulated residue, relates to a small $\epsilon$-approximation of the integrand in (3.6). The applied radiation condition is indirectly responsible for the Heaviside factor $H(x \Omega)=1 / 2+1 / 2 \operatorname{sgn} x \operatorname{sgn} \Omega$, with $\operatorname{sgn} x= \pm 1(x \gtrless 0)$.

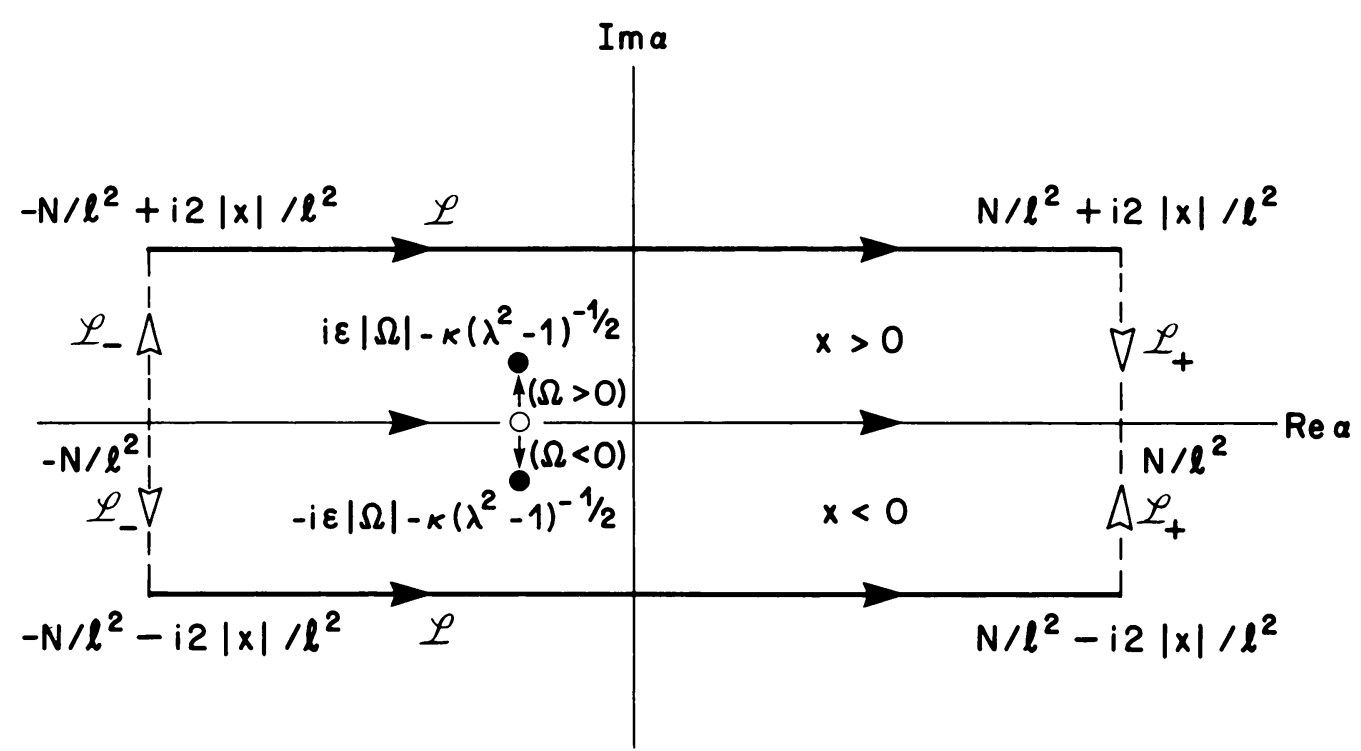

FIG. 1. Possible deformations of the path $\left(-N / l^{2}, N / l^{2}\right)$ into $\operatorname{Im} \alpha \gtrless 0$ depending on $x \gtrless 0$. The radiation condition causes the single pole to be slightly and vertically displaced from the (zero $\epsilon$ ) position $-\kappa\left(\lambda^{2}-1\right)^{-1 / 2}$ so that it contributes (or does not contribute) provided $x \Omega>0$ (or $<0$ ). Ultimately, there is just one other contribution, viz. an error term associated with either possible horizontal \&-path. 
Now

$$
\int_{\mathscr{L}}=\frac{\exp \left(-x^{2} / l^{2}\right)}{\left(\lambda^{2}-1\right)^{1 / 2}} \int_{-N / l 2}^{N / l^{2}} \frac{\exp \left(-\frac{1}{4} s^{2} l^{2}\right) d s}{s+\kappa\left(\lambda^{2}-1\right)^{-1 / 2}+i\left(2 x / l^{2}-\epsilon \Omega\right)}
$$

So,

$$
\begin{aligned}
\left(\lambda^{2}-1\right)^{1 / 2} & \exp \left(x^{2} / e^{2}\right)\left|\int_{\mathcal{L}}\right| \\
& \leq \int_{-N / l 2}^{N / l^{2}} \frac{|s|+\kappa\left(\lambda^{2}-1\right)^{-1 / 2}+\left|2 x / l^{2}-\epsilon \Omega\right|}{\left[s+\kappa\left(\lambda^{2}-1\right)^{-1 / 2}\right]^{2}+\left(2 x / l^{2}-\epsilon \Omega\right)^{2}} \exp \left(-\frac{1}{4} s^{2} l^{2}\right) d s \\
& <\frac{2}{\left(2 x / l^{2}-\epsilon \Omega\right)^{2}} \int_{0}^{N / l^{2}}\left[s+\kappa\left(\lambda^{2}-1\right)^{-1 / 2}+\left|2 x / l^{2}-\epsilon \Omega\right|\right] \exp \left(-\frac{1}{4} s^{2} l^{2}\right) d s \\
& =l\left(x-\frac{1}{2} \epsilon \Omega l^{2}\right)^{-2}\left\{l\left[1-\exp \left(-\frac{1}{4} N^{2} / l^{2}\right)\right]\right. \\
& \left.+\sqrt{ } \pi\left[\frac{1}{2} \kappa l^{2}\left(\lambda^{2}-1\right)^{-1 / 2}+\left|x-\frac{1}{2} \epsilon \Omega l^{2}\right|\right] \operatorname{erf}\left(\frac{1}{2} N / l\right)\right\} .
\end{aligned}
$$

Also, it can be shown that

$$
\left|\int_{L_{ \pm}}\right| \leq \frac{2|x| \exp \left(-\frac{1}{4} N^{2} / l^{2}\right)}{l^{2}\left(\lambda^{2}-1\right)^{1 / 2}} \int_{0}^{1} \frac{\exp \left[-s(2-s) x^{2} / l^{2}\right] d s}{\left| \pm N / l^{2}+\kappa\left(\lambda^{2}-1\right)^{-1 / 2}+i\left(2 x s / l^{2}-\epsilon \Omega\right)\right|} .
$$

It now remains to formulate the particular residue. This is found to contain the factor exp $(-\epsilon x \Omega)$ which, when coupled to $H(x \Omega)$ (see (3.13)), implies the existence of a slight artificial resistance reminiscent of that devised by Rayleigh (see e.g. [6], also [3]).

Let $N \rightarrow \infty$. From (3.16), then, $\int_{\mathscr{L}_{ \pm}} \rightarrow 0$. At this stage, in accordance with Lighthill, the limit, as $\epsilon \rightarrow 0$, may also be effected. Define the error term

$$
e(x \mid \kappa)=\lim _{\epsilon \rightarrow 0} \lim _{N \rightarrow \infty}(2 \pi)^{-1} \int_{\dot{L}}
$$

Whereupon, from (3.6) and (3.13), one eventually arrives at

$$
\lim _{\epsilon \rightarrow 0} \chi(x \mid \kappa)=i\left(\lambda^{2}-1\right)^{-1 / 2}(\operatorname{sgn} x) H(x \Omega) \exp \left[-i \kappa x\left(\lambda^{2}-1\right)^{-1 / 2}-\frac{1}{4} \kappa^{2} l^{2}\left(\lambda^{2}-1\right)^{-1}\right]+e(x \mid \kappa) \text {. }
$$

From (3.15),

$$
|e(x \mid \kappa)|<\frac{l \exp \left(-x^{2} / l^{2}\right)}{2 \pi x^{2}\left(\lambda^{2}-1\right)^{1 / 2}}\left\{l+\sqrt{ } \pi\left[\frac{1}{2} \kappa l^{2}\left(\lambda^{2}-1\right)^{-1 / 2}+|x|\right]\right\} .
$$

So far, much of the analysis relies upon $x \neq 0$.

Suppose $x=0$. Then (3.19) fails. However, (3.14) and (3.17) remain valid provided $l \neq$ 0 and reveal, by comparison with (3.6), that

$$
\lim _{\epsilon \rightarrow 0} \chi(0 \mid \kappa)=e(0 \mid \kappa) .
$$

If $l=0, \chi(0 \mid \kappa)$ can be explicitly determined from (3.6) under the approximation (3.7) by first deforming the $\alpha$-path $(-\infty, \infty)$ into $\operatorname{Im} \alpha \lessgtr 0$ whenever $\Omega \gtrless 0$, i.e. always opposite to the pole in (3.10), until the deformed path becomes an infinite semicircle. Along this, it is found that the integration does not vanish, but, via the Cauchy-Goursat theorem, in fact leads to

$$
\lim _{\epsilon \rightarrow 0} \chi(0 \mid \kappa)=\frac{1}{2} i\left(\lambda^{2}-1\right)^{-1 / 2} \operatorname{sgn} \Omega .
$$


4. The physical solution and its geometrical interpretation. In the steady (i.e. $\lim _{\epsilon \rightarrow 0}$ ) state,

$$
\Phi=\frac{H(x \Omega)}{2 \pi\left(\lambda^{2}-1\right)^{1 / 2}} \sum_{\nu=0}^{\infty} \frac{1}{\nu !}\left(\frac{1}{2} l \lambda\right)^{2 \nu} \frac{\partial^{2 \nu}}{\partial|x|^{2 \nu}} \int_{0}^{\infty} J_{0}(\kappa r) \sin \left[\kappa|x|\left(\lambda^{2}-1\right)^{-1 / 2}\right] d \kappa+E(x, r),
$$

where

$$
E(x, r)=(4 \pi)^{-1} \int_{0}^{\infty} J_{0}(\kappa r) \exp \left(-\frac{1}{4} \kappa^{2} l^{2}\right)[e(x \mid \kappa)-e(x \mid-\kappa)] d \kappa
$$

These are obtained by applying (3.18) to (3.5) and expanding a joint exponent factor uniformly over $0<\kappa<\infty$.

Results will now be presented for small $l$, small compared with $x(\neq 0)$. To estimate the quantity $E$, we first observe from the arguments leading to (3.19) that $|e(x \mid-\kappa)|$ satisfies the same inequality (i.e. (3.19)) as $|e(x \mid \kappa)|$, and then use the fact $[11, \S 2.5]$ that $\left|J_{0}(\kappa r)\right|$ $\leq 1$. Thereupon, it can be shown that

$$
|E(x, r)|<\exp \left(-x^{2} / l^{2}\right) \frac{l+l\left(\lambda^{2}-1\right)^{-1 / 2}+|x| \sqrt{ } \pi}{(2 x)^{2} \pi^{3 / 2}\left(\lambda^{2}-1\right)^{1 / 2}} .
$$

The $\kappa$-integral involved in (4.1) possesses the discontinuous value (cf. [11, §13.42])

$$
\left[x^{2}\left(\lambda^{2}-1\right)^{-1}-r^{2}\right]^{-1 / 2} H\left\{|x|\left(\lambda^{2}-1\right)^{-1 / 2}-r\right\} .
$$

Consequently, defining

$$
G(x, r)=(2 \pi)^{-1} \sum_{\nu=0}^{m-1} \frac{1}{\nu !}\left(\frac{1}{2} l \lambda\right)^{2 \nu} \frac{\partial^{2 \nu}}{\partial|x|^{2 \nu}}\left[x^{2}-r^{2}\left(\lambda^{2}-1\right)\right]^{-1 / 2}+O\left(l^{2 m}\right)
$$

for any finite positive integer $m$, one may express

$$
\begin{aligned}
\Phi & =G(x, r) \text { when } x \operatorname{sgn} \Omega>r\left(\lambda^{2}-1\right)^{1 / 2} \\
& =E(x, r) \text { when } x \operatorname{sgn} \Omega<r\left(\lambda^{2}-1\right)^{1 / 2},
\end{aligned}
$$

$E(x, r)$ being, at most, exponentially small like $x^{-1} \exp \left(-x^{2} / l^{2}\right)$.

Now the plane $x=0$, although excluded from the above discussion, forms part of the range $x$ sgn $\Omega<r\left(\lambda^{2}-1\right)^{1 / 2}$. On this plane, the result (4.7) remains formally consistent if $l$ $\neq 0$ in view of $(3.5),(3.20)$ and (4.2), equivalent to

$$
\left.\Phi\right|_{x=0}=E(0, r) .
$$

But the inequality (4.3) no longer holds; nonetheless, this does not imply an unbounded $\Phi$ field on $x=0$.

The solution comprising (4.6) and (4.7) is not essentially difficult to interpret. However, its implication goes deeper. To examine this, we first align the $x$-axis with the $\alpha$-axis of an $\alpha$-frame whose origin is located downstream (of the source's mean position) at $\mathbf{x}=$ $V$. Now introduce the unit wave vector $n=\alpha|\alpha|^{-1}=(\cos \phi, \sin \phi \cos \omega, \sin \phi \sin \omega)$ with $\phi$ $\in(0, \pi)$ and $\omega \in(\theta-\pi, \theta+\pi), \theta$ being the observational polar angle. Consider the equation

$$
\lambda^{2} \cos ^{2} \phi=1 \text {. }
$$

This is, equivalently, $\cot ^{2}(\phi-1 / 2 \pi)=\lambda^{2}-1$, implying that the two infinite circular cones

$$
C_{+}(x>0), C_{-}(x<0): \quad x^{2}=r^{2}\left(\lambda^{2}-1\right)
$$


covertexed at the mean distribution point $\mathbf{x}=0$ have their (spinning) generator orthogonal to the particular (spinning) direction $\mathrm{n}$ that is consistent with (4.9). Using (2.15) and remembering that $V \cos \phi=V \cdot n$, Eq. (4.9) can also be manipulated into the form

$$
(-\mathbf{V} \cdot \mathbf{n})^{4}-(-\mathbf{V} \cdot \mathbf{n})^{2}\left(a^{2}+c^{2}\right)+a^{2} c^{2} \cos ^{2} \phi=0,
$$

which becomes the familiar MGD dispersion relation [10] governing the phase speed $u(\mathrm{n})$ in the specific direction $\mathrm{n}$ after the substitution $-\mathrm{V} \cdot \mathrm{n}=u(\mathrm{n})$. This requires that the mean position $\alpha=-\mathrm{V}$ lie on the polar plane, with respect to the unit sphere $|\boldsymbol{\alpha}|=1$, of the particular point $\alpha=n / u(n)$ which, in turn, lies on a normal surface. (The present argument is comparable to that of [1] for the two-dimensional but generalized system.) Now, fundamental MGD waves are transversely isotropic and non-dispersive. Such waves have their phase speed and group velocity $U$ related, along any direction $n$, by [2]

$$
U=n\left[u(\mathrm{n})-\mathrm{n} \cdot \nabla_{n} u(\mathrm{n})\right]+\nabla_{n} u(\mathrm{n})
$$

with $\nabla_{n}=(\partial / \partial(\cos \phi), \partial / \partial(\sin \phi \cos \omega), \partial / \partial(\sin \phi \sin \omega))$. So $\mathbf{U} \cdot \mathbf{n}=u(\mathbf{n}) \forall \mathbf{n}$ (see also [7]); i.e., the $U$-surface $\alpha=U$ is an envelope of polar planes, with respect to $|\boldsymbol{\alpha}|=1$, of all points on the normal surface; $\alpha=n / u(n)$. Thus, whenever a real tangent cone to the $U$ surface can be vertexed at $\alpha=-\mathrm{V}$ (i.e. at $\mathbf{x}=0$ ), (4.9) admits at least one real $\mathrm{n}$-root orthogonal to its generator; here, $n$ satisfies $(\mathbf{V}+\mathbf{U}) \cdot \mathbf{n}=0$ (see Fig. 2). Evidently, this tangent cone has the equation (4.10). It is actually identical to $C_{+}$. As yet, there is no special mathematical reason why the origin $\alpha=0$ should not be taken upstream at $\mathbf{x}=$ $-\mathrm{V}$; if this is indeed the choice, one would eventually arrive at a tangent cone identifiable as $C_{-}$.

Suppose $M>1$ and $A>1$, i.e. $V>\max (a, c)$. This covers $M=3, A=4$, for which certain computations are available in [4]. Unfortunately, the attention Crapper paid to this particular line is somewhat cursory; as such, the information provided by his asymptotic results does not adequately account for the detailed overall picture; moreover, by focussing on the far field, the important behavior off the cone surface $C_{+}$, under investigation throughout this section, has been unfortunately overlooked. Relative to the MGD U-surface or Friedrichs wavefront (a surface of revolution), the point $\alpha=-\mathrm{V}$ is outside the "fast" sheet which cuts the $\alpha$-axis (of symmetry) at $\alpha=\max (a, c),-\max (a$, $c)$. In this event, we obtain a downstream tangent cone $C_{+}$plus an upstream cone $C_{-}$ covertexed at $\alpha=-V$. But then $\Omega>0$ (see Sec. 3). This allows only the actual participation of the downstream tangent cone $C_{+}$and excludes that of the upstream cone $C_{-}$. In fact, (4.6) and (4.7) disclose that the $\Phi$-field is determined by

$$
\begin{aligned}
& G(x, r) \quad \text { (strong field) downstream of } C_{+}, \\
& E(x, r) \quad \text { (weak field) upstream of } C_{+} .
\end{aligned}
$$

The radiation scheme is portrayed in Fig. 2.

Crapper's analysis [4] avoids the alternate situation: $M<1, A<1$ but $M^{2}+A^{2}>1$. Here, $a c\left(a^{2}+c^{2}\right)^{-1 / 2}<V<\min (a, c)$, so that the mean position $\alpha=-\mathrm{V}$ terminates inside the left member of the "slow" cusped U-sheets which cuts the $\alpha$-axis at $\alpha=-a c\left(a^{2}+\right.$ $\left.c^{2}\right)^{-1 / 2}$ and again at $\alpha=-\min (a, c)$. Of both covertexed cones representable again by (4.10), the upstream cone $C_{\text {- }}$ is tangential to the left cusped $U$-sheet (cf. [5]. Note that the present $\left(C_{+}, C_{-}\right)$pair of cones is totally distinct from the previous $\left(C_{+}, C_{-}\right)$pair in the case $M>1, A>1$ ). Since $\Omega<0$ now, the $\Phi$-field must be interpreted from (4.6) and (4.7) as being 


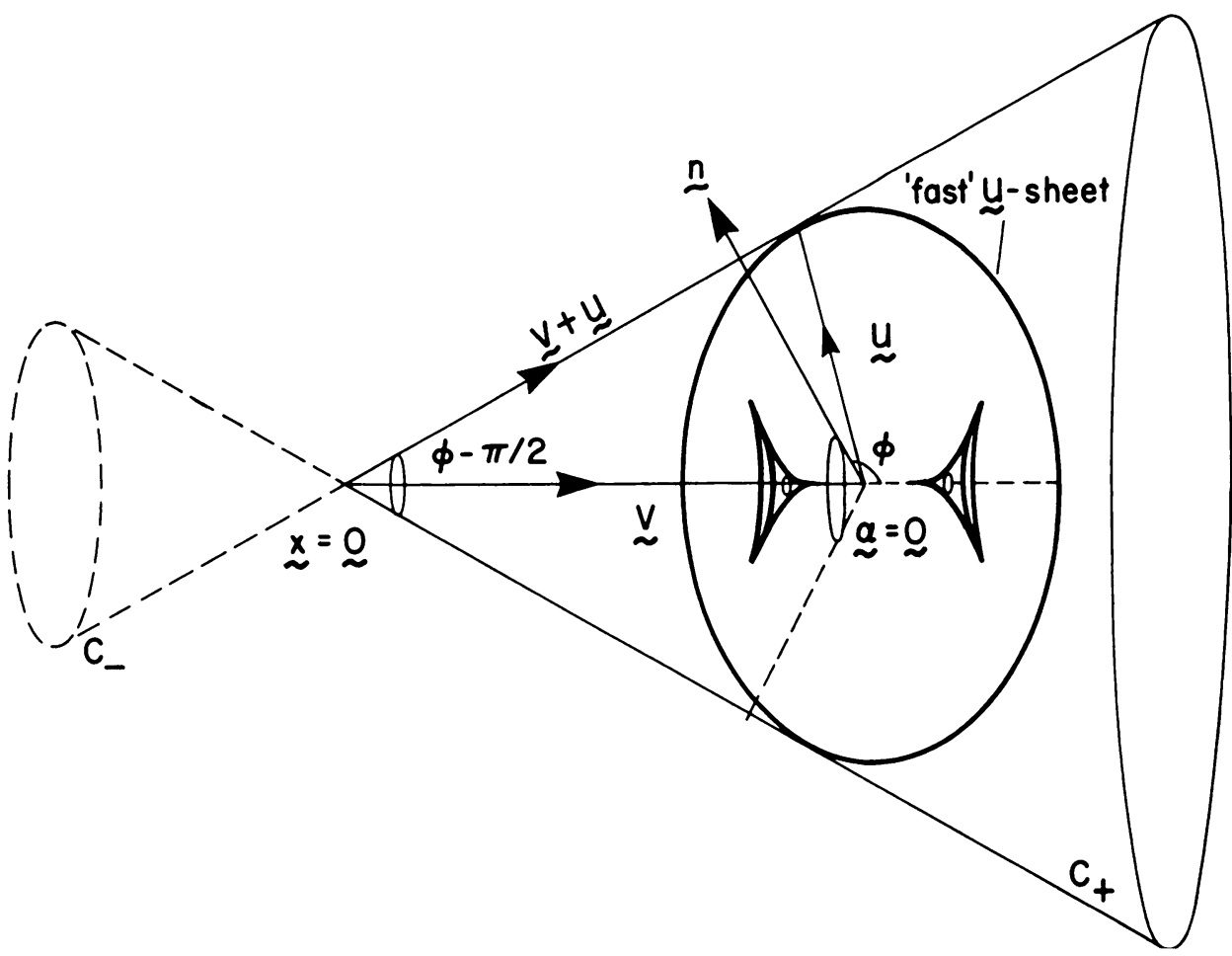

FIG. 2. The construction of the conical $\left(C_{+}, C_{-}\right)$pair as a simultaneous envelopment of just that subset of relevant polar planes passing through the mean distribution point $\mathbf{x}=0$. The complete set of polar planes envelope the $\mathrm{U}$-surface. The MGD flow regime here corresponds to $V>\max (a, c)$, i.e. supersonic-superAlfvénic. In this case, the downstream cone $C_{+}$is tangential to the "fast" sheet of the Friedrichs U-surface (cf. [10]) and, via an applied radiation condition, separates the emission into a strong $G$-field downstream and a weak $E$-field upstream. This radiation condition totally excludes the physical presence of the complementary upstream cone $C_{-}$.

$$
\begin{aligned}
& G(x, r) \quad \text { (strong field) upstream of } C_{-}, \\
& E(x, r) \quad \text { (weak field) downstream of } C_{-},
\end{aligned}
$$

and, obviously, never involves the downstream cone $C_{+}$. Fig. 3 depicts the radiation scheme.

In each instance the weak $E$-field occupies the larger space external to a cone and including the plane $x=0$ (see (4.8)). From (4.3), $E \rightarrow 0$ as $l \rightarrow 0$ provided that $x \neq 0$. To accommodate $x=0$ in the limit, we note that the right side of (3.21) is independent of $\kappa$ so that, by (3.5), $\left.\Phi\right|_{x=0} \equiv 0$ when $l=0$, in which case: $\Phi \equiv 0$ (everywhere) upstream of $C_{+}$if $V>\max (a, c)$, or downstream of $C_{\text {- }}$ if $a c\left(a^{2}+c^{2}\right)^{-1 / 2}<V<\min (a, c)$; moreover $\Phi=$ $G(x, r) \equiv(2 \pi)^{-1}\left[x^{2}-r^{2}\left(\lambda^{2}-1\right)\right]^{-1 / 2}$ across the particular cone. This solution represents the Green's function (i.e. the point source potential), and is analogous to that for nonmagnetic supersonic flow. In fact, the propagation pattern for $V>\max (a, c)$ and $l=0$ may be deduced from such an analogy [5, 10]; here, the geometrical connection arises, via a Huygens-type principle, from a physically-based envelopment of successive elementary perturbations. Whilst this method does intuitively identify $C_{-}$as the physical envelope when $a c\left(a^{2}+c^{2}\right)^{-1 / 2}<V<\min (a, c)$, it cannot convincingly tell us to which side of $C_{-}$ 
the significant $G$-field is confined. In contrast, the only physical notion we have introduced is the well-tried and much more fundamental Lighthill version of the radiation condition. Combined with Fourier and geometrical constructions, it leads to the unique solution (see $[7,8,9])$ with supporting conical domains clearly defined.

The strong $G$-field (for $l \neq 0$ ) can be asymptotically expanded along any fixed radial line passing from the vertex $\mathbf{x}=0$ into the supporting conical interior $x$ sgn $\Omega>$ $r\left(\lambda^{2}-1\right)^{1 / 2}$. Thus, if $\psi$ denotes the angular colatitude of a spherical polar frame with radial distance $|\mathbf{x}|: x=|\mathbf{x}| \cos \psi$ while $r=|\mathbf{x}| \sin \psi$, then for fixed $\psi$ and within the specified interior, (4.5) can be shown to develop into

$$
G(x, r)=(2 \pi)^{-1}(\operatorname{sgn} \Omega) \sum_{\nu=0}^{m-1} \frac{B_{\nu}(\sec \psi)^{2 \nu+1}(l \lambda)^{2 \nu}}{4^{\nu} \nu !|\mathbf{x}|^{2 \nu+1}}+O\left(l^{2 m} /|\mathbf{x}|^{2 m+1}\right)
$$

where, in terms of coefficients $A_{\mu}=2^{-2 \mu}(\mu !)^{-2}(2 \mu) !(\mu=0,1, \ldots, \infty)$ in the binomial expansion of $(1-z)^{-1 / 2}$ within $-1 \leq z<1$, the coefficient

$$
B_{\nu}=\sum_{\mu=0}^{\infty} A_{\mu}(\tan \psi)^{2 \mu}\left(\lambda^{2}-1\right)^{\mu}(2 \mu+2 \nu) ! /(2 \mu) ! .
$$

For sufficiently large $|\mathbf{x}|$, the expanded form (4.15) is dominated by its leading term, which

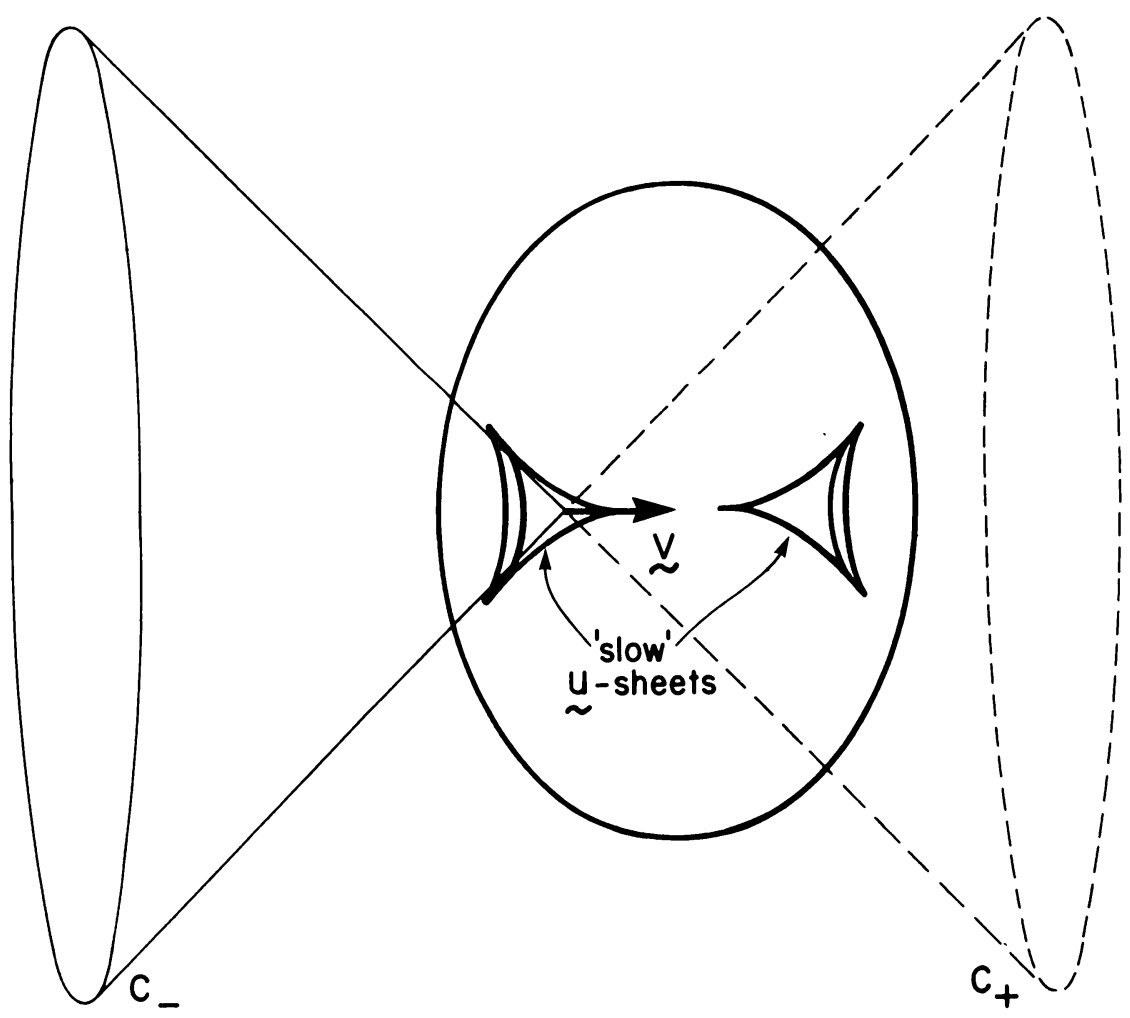

FIG. 3. The $a c\left(a^{2}+c^{2}\right)^{-1 / 2}<V<\min (a, c)$ (i.e. restricted subsonic-subAlfvénic) flow configuration. Of the $\left(C_{+}, C_{-}\right)$pair, the $C_{+}$member is physically inadmissible according to the radiation condition which, in turn, restricts the strong $G$-field upstream (or weak $E$-field downstream) of $C_{-}$, the tangent cone from the point $\mathbf{x}=0$ to the surrounding "slow" cusped U-sheet. 
is of order $|\mathbf{x}|^{-1}$, and to which $G(x, r)$ degenerates when $l=0$ (cf. the preceding paragraph). Other asymptotic modes will be demonstrated next in Sec. 5.

5. A uniformly valid velocity gradient. So far, approximate results hold only for small $l(\geq 0)$, and for observations off either cone $C_{+}$or $C_{-}$, whichever is relevant. A uniformly valid solution, applicable along $C_{+}$and $C_{-}$, is available provided $l \neq 0$, assumed throughout this section. It is convenient here to work with a perturbation gradient because, then, derivatives of $\Phi$ (rather than $\Phi$ itself) are involved. For this purpose, we define the quantity

$$
\Gamma=2 \pi\left(A^{2}-\lambda^{2}\right)^{-1}\left(M^{2}+A^{2}-1\right)\left(\lambda^{2}-1\right)^{1 / 2} \partial v_{1} / \partial x,
$$

which is proportional to the $x$-gradient of the $x$-velocity component $v_{1}$. This also expedites comparison with Crapper's results [4] based on $\partial v_{1} / \partial x$ (which, incidentally, is Crapper's " $\Gamma$ ").

The desired $\Gamma$-solution is then fully described by the following expressions (5.2) - (5.5) (whose derivations are deferred to an appendix):

$$
\begin{aligned}
\Gamma=\Delta+2 \pi\left(\lambda^{2}-1\right)^{-1 / 2}\left\{\left(1-A^{2}\right)\left(A^{2}-\lambda^{2}\right)^{-1}(l \sqrt{ } \pi)^{-3}\right. & \exp \left(-\mathbf{x}^{2} / l^{2}\right) \\
- & \left.\left(\partial^{2} / \partial r^{2}+r^{-1} \partial / \partial r\right) E(x, r)\right\}
\end{aligned}
$$

where

$$
\begin{aligned}
\Delta=-\frac{1}{2}(\pi r)^{-1 / 2}(\operatorname{sgn} \Omega) H( & x \Omega)\left\{\frac{\partial^{2}}{\partial x^{2}}\left(F_{+}+F_{-}\right)\right. \\
+ & \left.\frac{\mu_{2}}{8 r\left(\lambda^{2}-1\right)^{1 / 2}} \frac{\partial}{\partial x}\left(F_{+}-F_{-}\right)+\frac{9 \mu_{1}\left(F_{+}+F_{-}\right)}{128 r^{2}\left(\lambda^{2}-1\right)}\right\},
\end{aligned}
$$

$\mu_{1}, \mu_{2}$ being Stieltjes constants for the Bessel function $J_{0}$ (see (A7)); also, in terms of

$$
\begin{gathered}
Z_{ \pm}{ }^{1 / 4}=2^{-1 / 4}(l \lambda)^{-1 / 2}\left(\lambda^{2}-1\right)^{1 / 4}\left|x\left(\lambda^{2}-1\right)^{-1 / 2} \pm r\right|^{1 / 2} \\
F_{ \pm}=\pi 2^{-1 / 4}(l \lambda)^{-1 / 2}\left(\lambda^{2}-1\right)^{1 / 4} \exp \left(-Z_{ \pm}\right) Z_{ \pm}{ }^{1 / 4}\left\{I_{1 / 4}\left(Z_{ \pm}\right) \operatorname{sgn}\left(x \pm r\left(\lambda^{2}-1\right)^{1 / 2}\right)\right. \\
\left.\mp I_{-1 / 4}\left(Z_{ \pm}\right)\right\}
\end{gathered}
$$

and whose $x$-derivatives appear in (5.3). To determine these, we need, for the modified Bessel function $I_{\nu}\left(Z_{ \pm}\right)$, the fact $Z_{ \pm}{ }^{\nu} I_{\nu}\left(Z_{ \pm}\right)=0$ whenever $Z_{ \pm}=0$ for $\nu=1 / 43 / 4$, together with the recurrence relations $[11, \S 3.71]$

$$
\frac{d}{d z}\left[z^{\nu} I_{\nu}(z)\right]=z^{\nu} I_{\nu-1}(z), \quad \frac{d}{d z}\left[z^{\nu} I_{-\nu}(z)\right]=z^{\nu} I_{-\nu+1}(z) .
$$

Thus,

$$
\begin{aligned}
& \partial F_{ \pm} / \partial x+(l \lambda)^{-2}\left(x \pm r\left(\lambda^{2}-1\right)^{1 / 2}\right) F_{ \pm} \\
& \begin{array}{c}
=\pi(l \lambda)^{-3 / 2} 2^{1 / 4}\left(\lambda^{2}-1\right)^{1 / 4} \exp \left(-Z_{ \pm}\right) Z_{ \pm}{ }^{3 / 4}\left\{I_{-3 / 4}\left(Z_{ \pm}\right)\right. \\
\left.\mp I_{3 / 4}\left(Z_{ \pm}\right) \operatorname{sgn}\left(x \pm r\left(\lambda^{2}-1\right)^{1 / 2}\right)\right\}, \\
\partial^{2} F_{ \pm} / \partial x^{2}+2(l \lambda)^{-2}\left(x \pm r\left(\lambda^{2}-1\right)^{1 / 2}\right) \partial F_{ \pm} / \partial x+(l \lambda)^{-2} F_{ \pm}=0 .
\end{array}
\end{aligned}
$$


The function $\Delta$ may now be computed from (5.3) - (5.7).

Additionally, (5.2) involves

$$
\left(\partial^{2} / \partial r^{2}+r^{-1} \partial / \partial r\right) E(x, r)=(4 \pi)^{-1} \int_{0}^{\infty} \kappa^{2} J_{0}(\kappa r) \exp \left(-\frac{1}{4} \kappa^{2} l^{2}\right)[e(x \mid-\kappa)-e(x \mid \kappa)] d \kappa,
$$

obtained from (4.2) and the Bessel equation

$$
J_{0}^{\prime \prime}(\kappa r)+(\kappa r)^{-1} J_{0}{ }^{\prime}(\kappa r)+J_{0}(\kappa r)=0 .
$$

As usual, it is complicated to evaluate an integral of the type (5.8) through the representation (3.17). However, as in (4.3), an upper bound can be similarly formulated via (3.19) if $x \neq 0$ :

$$
\left|\left(\partial^{2} / \partial r^{2}+r^{-1} \partial / \partial r\right) E(x, r)\right|<\exp \left(-x^{2} / l^{2}\right) \frac{1+2\left(\lambda^{2}-1\right)^{-1 / 2}+\sqrt{ }|x| / l}{2 x^{2} \pi^{3 / 2} l\left(\lambda^{2}-1\right)^{1 / 2}} .
$$

Now we examine certain aspects of the asymptotic development while $|x| \rightarrow \infty$. Unless otherwise specified, $l$ is assumed to be finite. Now, when $\operatorname{sgn}\left(x \pm r\left(\lambda^{2}-1\right)^{1 / 2}\right)= \pm 1$, the right sides of (5.5) and (5.6) are associated with the function

$$
\begin{aligned}
\exp \left(-Z_{ \pm}\right) Z_{ \pm}{ }^{\nu}\left[I_{-\nu}\left(Z_{ \pm}\right)-\right. & \left.I_{\nu}\left(Z_{ \pm}\right)\right]=(2 / \pi) \sin (\nu \pi) \exp \left(-Z_{ \pm}\right) Z_{ \pm}{ }^{\nu} K_{\nu}\left(Z_{ \pm}\right) \\
& \sim(2 / \pi)^{1 / 2} \sin (\nu \pi) \exp \left(-2 Z_{ \pm}\right) Z_{ \pm}{ }^{\nu-1 / 2}
\end{aligned}
$$

as $x \rightarrow \pm \infty$ (cf. $[11, \S \S 3.7,7.23)$, in which case $\partial^{\mu} F_{ \pm} / \partial x^{\mu}(\mu=0,1,2)$ diminish exponentially by virtue of (5.4) - (5.7). Hence, omitting known small terms, (5.2) and (5.3) reduce to:

$$
\begin{aligned}
& \Gamma \sim \frac{-H(x)}{2(\pi r)^{1 / 2}}\left\{\frac{\partial^{2} F_{-}}{\partial x^{2}}-\frac{\mu_{2}}{8 r\left(\lambda^{2}-1\right)^{1 / 2}} \frac{\partial F_{-}}{\partial x}+\frac{9 \mu_{1} F_{-}}{128 r^{2}\left(\lambda^{2}-1\right)}\right\} \quad \text { if } \quad \Omega>0, \\
& \Gamma \sim \frac{H(-x)}{2(\pi r)^{1 / 2}}\left\{\frac{\partial^{2} F_{+}}{\partial x^{2}}+\frac{\mu_{2}}{8 r\left(\lambda^{2}-1\right)^{1 / 2}} \frac{\partial F_{+}}{\partial x}+\frac{9 \mu_{1} F_{+}}{128 r^{2}\left(\lambda^{2}-1\right)}\right\} \quad \text { if } \quad \Omega<0 .
\end{aligned}
$$

While we now know that, relative to the plane $x=0, \Gamma$ is negligible far upstream (or downstream) whenever $\Omega<0$, we have yet to learn explicitly just how significant it is far downstream (or upstream), especially with reference to the cone $C_{ \pm}: x= \pm r\left(\lambda^{2}-1\right)^{1 / 2}$ ).

From (5.5) and the $I_{\nu}$-series,

$$
F_{\mp}= \pm \frac{\pi\left(\lambda^{2}-1\right)^{1 / 4}}{(I \lambda)^{1 / 2} \Gamma(3 / 4)}+\frac{\pi\left(\lambda^{2}-1\right)^{1 / 4}\left(x \mp r\left(\lambda^{2}-1\right)^{1 / 2}\right)}{2(l \lambda)^{3 / 2} \Gamma(5 / 4)}+O\left\{\left(x \mp r\left(\lambda^{2}-1\right)^{1 / 2}\right)^{2}\right\}
$$

when $x \approx \pm r\left(\lambda^{2}-1\right)^{1 / 2}$, so that from (5.6),

$$
\frac{\partial F_{\mp}}{\partial x}=\frac{2 \pi\left(\lambda^{2}-1\right)^{1 / 4}}{(l \lambda)^{3 / 2} \Gamma(1 / 4)}+O\left(x \mp r\left(\lambda^{2}-1\right)^{1 / 2}\right) .
$$

Suppose, according as $\Omega<0$, the reception point $\mathbf{x}$ is fixed near the existing cone $C_{ \pm}$in $x<$ 0 but stays far enough from its vertex:

$$
|x|^{-1}=o\left(\left|x \mp r\left(\lambda^{2}-1\right)^{1 / 2}\right|\right) .
$$

Whereupon, after estimating $\partial^{2} F_{\mp} / \partial x^{2}$ via (5.7), (5.14), (5.15) and defining 


$$
\Gamma_{0}=\frac{1}{2}(\pi / r)^{1 / 2}\left(\lambda^{2}-1\right)^{1 / 4} /(l \lambda)^{5 / 2} \Gamma(3 / 4),
$$

it follows from (5.12) and (5.13) that

$$
\Gamma \sim \Gamma_{0} \pm 6 \Gamma_{0}(l \lambda)^{-1}\left(x \mp r\left(\lambda^{2}-1\right)^{1 / 2}\right) \Gamma(3 / 4) / \Gamma(1 / 4) .
$$

This result, obviously nonsingular, holds far away along $C_{ \pm}$itself: $\Gamma \sim \Gamma_{0}$, which is proportional to $r^{-1 / 2} l^{-5 / 2}$. This confirms Crapper's deduction [4] in the case $\Omega>0$. Although Crapper did not provide an explicit form of the amplitude, this can be remedied via geometrical techniques. Now, corresponding to three co-cylindrical positions, one of which lies on $C_{ \pm}$whilst the other two are near and on opposing sides of it but remain governed by $(5.16)$, we see from $(5.18)$ that relative to $C_{+}$:

$$
\Gamma_{\text {downstream }}>\Gamma_{0}>\Gamma_{\text {upstream }}(\Omega>0) \text {, }
$$

whilst relative to $C_{-}$:

$$
\Gamma_{\text {downstream }}<\Gamma_{0}<\Gamma_{\text {upstream }}(\Omega<0) .
$$

Thus, consistent with our deductions in Sec. 4 concerning $\Phi$, the stronger far-near field occurs downstream of $C_{+}$when $\Omega>0$, or upstream of $C_{-}$when $\Omega<0$. Nevertheless, in the present situation with finite $l$, the far-near fields on both sides of $C_{ \pm}(\Omega<0)$ are of comparable intensities. In contrast, our earlier $\Phi$-solution for small $l$ discloses that the stronger field to one side of $C_{ \pm}$is considerably stronger than the adjacent weaker field, the latter being weaker than a known exponentially small quantity; in fact, this weaker field is further weakened towards zero as $|x| \rightarrow \infty$. These factors suggest the possible existence of a conical $C_{ \pm}$-layer virtually sheathing $C_{ \pm}$and within which the field changes gradually. In the case of moderate $l$, such a $C_{ \pm}$-layer is detectable, at least asymptotically, as already experienced through the function $\Gamma$. But if $l$ is small (approaching zero, say), then this $C_{ \pm}$layer is thin (shrinking onto $C_{ \pm}$), and one suspects that our earlier $\Phi$-results hold onily outside such a thin layer; this would then explain the sudden discontinuity across $C_{ \pm}$.

Next, suppose $\mathbf{x}$ is far from and between both the plane $x=0$ and the cone $C_{ \pm}(\Omega<0)$. The approximations (5.12) and (5.13) are again valid. Furthermore, $x \mp r\left(\lambda^{2}-1\right)^{1 / 2}>0$ and is large, in which event (5.11) also applies and reveals $\partial^{\mu} F_{\mp} / \partial x^{\mu}(\mu=0,1,2)$ to be exponentially small. Consequently $\Gamma \sim 0$, which now holds far from either side of the plane $x=0$ but far upstream of $C_{+}$when $\Omega>0$ (or far downstream of $C_{-}$when $\Omega<0$ ), as well. Such an observation should be well outside any actual $C_{+}\left(C_{-}\right)$layer, and we do have some compatibility with the weak $E$-representation in (4.12) (or (4.14)). In fact, the same conclusion can be arrived by substituting "at some finite distance" for "far" and assuming a small $l$, since then $Z_{\mp}$ remains large whilst $(5.11)-(5.13)$ still hold.

Suppose, on the other hand, $\mathbf{x}$ is reasonably far downstream of $C_{+}$when $\Omega>0$ (or far upstream of $C_{-}$when $\Omega<0$ ) and even very much further off from the $x$-axis, viz such that $r\left(\lambda^{2}-1\right)^{1 / 2} \gg \mid x-($ or +$) r\left(\lambda^{2}-1\right)^{1 / 2} \mid$ which is appreciably large. First, since sgn $(x \mp$ $\left.r\left(\lambda^{2}-1\right)^{1 / 2}\right)= \pm 1$, the corresponding right sides of (5.5) and (5.6) for $F_{\mp}, \partial F_{\mp} / \partial x$ now involve (contrast $(5.11)$; cf. [11, §7.23])

$$
\begin{aligned}
\exp \left(-Z_{\mp}\right) Z_{\mp}{ }^{\nu}\left[I_{-\nu}\left(Z_{\mp}\right)+I_{\nu}\left(Z_{\mp}\right)\right] \sim\left(\frac{2}{\pi}\right)^{1 / 2} & Z_{\mp}{ }^{\nu-1 / 2} \\
& \times\left[1+\frac{1-4 \nu^{2}}{8 Z_{\mp}}+\frac{\left(1-4 \nu^{2}\right)\left(9-4 \nu^{2}\right)}{128 Z_{\mp}{ }^{2}}+\cdots\right] .
\end{aligned}
$$


It may then be verified from (5.4) - (5.7) plus (5.12) and (5.13) that

$$
\Gamma \sim-3\left(\lambda^{2}-1\right)^{1 / 4} r^{-1 / 2}\left(2 \mid x-(\text { or }+) r\left(\lambda^{2}-1\right)^{1 / 2} \mid\right)^{-5 / 2}
$$

This far-far field, though overwhelmingly stronger than its negligible counterpart across $C_{+}$(or $C_{-}$), is still weak in comparison with the far-near field on the same side, i.e. $\Gamma_{\text {downstream }}$ of (5.19) (or $\Gamma_{\text {upstream }}$ of (5.20)).

Crapper's [4] stationary-phase technique led him to conclude that the $\Gamma$-disturbance is asymptotically confined to the cone $C_{+}$whenever $\Omega>0$. We now know that this is not absolutely true. However, our analysis so far appears to support only one possibility, viz. that the bulk of the $\Gamma$-disturbance is concentrated about $C_{ \pm}(\Omega<0)$, probably within the $C_{ \pm}$-layer conjectured earlier on. The issue can be decided only after the relative far-field strength at any distance from $C_{ \pm}$has been rigorously explored for finite $l$.

Appendix. Expressions pertaining to a velocity gradient. The objective here is the establishment of the steady-state expressions (5.2) - (5.5) accompanying definition (5.1). This involves the velocity gradient $\partial v_{1} / \partial x$ which must first be linked with the $\Phi$-field. Applying liberally the solenoidal condition $\nabla \cdot \mathrm{h}=0$ of (2.2), the induction equation in (2.1) simplifies to

$$
\partial \mathrm{v} / \partial x-V \partial \mathrm{h} / \partial x-(\nabla \cdot \mathrm{v}, 0,0)=\partial \mathrm{h} / \partial t=0
$$

during the steady state, so that on appealing to $(2.4)$, we get

$$
\left(M^{2}+A^{2}-1\right) \partial \mathbf{v} / \partial x=(\nabla \cdot \mathbf{Q}, 0,0)-A^{2} \partial \mathbf{Q} / \partial x .
$$

Consequently, via (3.1),

$$
\left(M^{2}+A^{2}-1\right) \partial v_{1} / \partial x=\nabla^{2} \Phi-A^{2} \partial^{2} \Phi / \partial x^{2} .
$$

In preference to (4.1), one can write, via (3.5) and (3.18),

$$
\Phi=(2 \pi)^{-1}\left(\lambda^{2}-1\right)^{-1 / 2}(\operatorname{sgn} \Omega) H(x \Omega) F(x, r)+E(x, r),
$$

with

$$
F(x, r)=F=\int_{0}^{\infty} J_{0}(\kappa r) \sin \left[\kappa x\left(\lambda^{2}-1\right)^{-1 / 2}\right] \exp \left[-\kappa^{2} l^{2} \lambda^{2} / 4\left(\lambda^{2}-1\right)\right] d \kappa
$$

and $E(x, r)$ given by (4.2). Application of (5.9) to (A2) and (A3) reveals that

$$
\begin{aligned}
& {\left[\partial^{2} / \partial r^{2}+r^{-1} \partial / \partial r+\left(1-\lambda^{2}\right) \partial^{2} / \partial x^{2}\right] \Phi} \\
& =\left[\partial^{2} / \partial r^{2}+r^{-1} \partial / \partial r+\left(1-\lambda^{2}\right) \partial^{2} / \partial x^{2}\right] E(x, r) \\
& \quad-(2 \pi)^{-1}\left(\lambda^{2}-1\right)^{1 / 2} \delta(x)(\partial F / \partial x)_{x=0},
\end{aligned}
$$

$\delta(x)$ being the one-dimensional Dirac delta function. But (2.16) and (3.1) imply that

$$
\left[\alpha^{2}-\alpha^{2} \lambda^{2}\left(V-i \epsilon \alpha^{-1}\right)\right] \hat{\Phi}=\hat{S}(\alpha),
$$

whose Fourier inverse, on approaching the steady state, ultimately reduces to

$$
\left[\partial^{2} / \partial r^{2}+r^{-1} \partial / \partial r+\left(1-\lambda^{2}\right) \partial^{2} / \partial x^{2}\right] \Phi=-S(\mathbf{x})=-(l \sqrt{ } \pi)^{-3} \exp \left(-\mathbf{x}^{2} / l^{2}\right),
$$

with $\lambda=\lambda(V)$. From this (A1), (A2), (A4) and (A5) can be shown to lead to the formula 
(5.2) for the quantity defined by (5.1), provided we have

$$
\Delta=-(\operatorname{sgn} \Omega) H(x \Omega) \partial^{2} F / \partial x^{2} .
$$

To exploit (A3) in (A6), it is desirable to write, in accordance with Stieltjes [11, $\$ 7.3$, $7.31]$

$$
J_{0}(\kappa r)=\left(\frac{2}{\pi \kappa r}\right)^{1 / 2}\left\{\left(1-\frac{9 \mu_{1}}{128 \kappa^{2} r^{2}}\right) \cos \left(\kappa r-\frac{1}{4} \pi\right)+\frac{\mu_{2}}{8 \kappa r} \sin \left(\kappa r-\frac{1}{4} \pi\right)\right\},
$$

$\mu_{1}$ and $\mu_{2}$ being positive constants less than one. Formula (5.3) is then derived, wherein it is first observed that

$$
\begin{aligned}
F_{ \pm} & =F_{ \pm}(x, r)=\sqrt{2} \int_{0}^{\infty} \sin \left\{\kappa\left[x\left(\lambda^{2}-1\right)^{-1 / 2} \pm r\right]^{1} \mp \frac{1}{4} \pi\right\} \exp \left[-\kappa^{2} l^{2} \lambda^{2} / 4\left(\lambda^{2}-1\right)\right] \kappa^{-1 / 2} d \kappa \\
& =\left[\frac{1}{2} \pi\left|x\left(\lambda^{2}-1\right)^{-1 / 2} \pm r\right|\right]^{1 / 2} \int_{0}^{\infty} \exp \left[-\kappa^{2} l^{2} \lambda^{2} / 4\left(\lambda^{2}-1\right)\right] \\
& \times\left\{J_{1 / 2}\left[\kappa\left|x\left(\lambda^{2}-1\right)^{-1 / 2} \pm r\right|\right] \operatorname{sgn}\left[x\left(\lambda^{2}-1\right)^{-1 / 2} \pm r\right] \operatorname{sgn}\left[x\left(\lambda^{2}-1\right)^{-1 / 2} \pm r\right]\right. \\
& \left.\mp J_{-1 / 2}\left[\kappa\left|x\left(\lambda^{2}-1\right)^{-1 / 2} \pm r\right|\right]\right\} d \kappa,
\end{aligned}
$$

after interpreting a sine and a cosine via the Bessel functions $J_{1 / 2}, J_{-1 / 2}[11, \S 3.4]$. The result $[11, \S 13.3]$

$$
\int_{0}^{\infty} J_{2 \nu}(\kappa|x|) \exp \left(-\kappa^{2} t^{2}\right) d \kappa=\frac{1}{2} v \pi t^{-1} \exp \left(-x^{2} / 8 t^{2}\right) I_{\nu}\left(x^{2} / 8 t^{2}\right)
$$

may now be applied to (A8) for $\nu=1 / 4,-1 / 4$, whereupon (5.5) follows.

\section{REFERENCES}

[1] L. Chee-Seng, Q.J. Mech. Appl. Math. 26, 371 (1973)

[2] L. Chee-Seng, Proc. Camb. Phil. Soc. 74, 369 (1973)

[3] G. D. Crapper, J. Fluid Mech. 6, 51 (1959)

[4] G. D. Crapper, J. Inst. Math. Applics. 1, 241 (1965)

[5] E. Cumberbatch, J. Aerospace Sci. 29, 1476 (1962)

[6] H. Lamb, Hydrodynamics, 6th ed., Cambridge University Press, 1932

[7] M. J. Lighthill, Phil. Trans. Roy. Soc. London A252, 397 (1960)

[8] M. J. Lighthill, J. Inst. Math. Applics. 1, 1 (1965)

[9] M. J. Lighthill, J. Fluid Mech. 27, 725 (1967)

[10] W. R. Sears and E. L. Resler, Advances in Applied Mechanics 8, Academic Press, New York, 1964

[11] G. N. Watson, Theory of Bessel functions, 2nd ed. Cambridge University Press, 1944 (reissued in paperback, 1966) 